\title{
SHEAVES WITH FINITELY GENERATED ISOMORPHIC STALKS AND HOMOLOGY MANIFOLDS
}

\author{
JERZY DYDAK AND JOHN WALSH
}

(Communicated by Doug W. Curtis)

\begin{abstract}
The setting is sneaves of modules over a commutative ring $L$. It is shown that on completely metrizable spaces certain sheaves having mutually isomorphic finitely generated stalks are locally constant over a dense open subset. This is used to show that a locally compact metrizable space $X$ that is homologically locally connected with respect to a principal ideal domain $L$ is a homology manifold over $L$ provided it has finite cohomological dimension with respect to $L$ and, for any two points $x, y \in X$, the modules $H_{k}(X, X-\{x\} ; L)$ and $H_{k}(X, X-\{y\} ; L)$ are isomorphic and finitely generated.
\end{abstract}

The purpose of this note is to provide a generalization of results of Bredon [ $\left.\mathbf{B}_{\mathbf{1}}\right]$ and Bryant $[\mathbf{B r}]$ that show under certain circumstances that homogeneous spaces are homology manifolds. In $\left[\mathbf{B}_{\mathbf{1}}\right]$ it is established, for a countable principal ideal domain $L$, that a locally compact metrizable space $X$ that is homologically locally connected with respect to $L$ and has finite cohomological dimension with respect to $L$ is a homology manifold over $L$ provided, for each pair of points $x, y \in X$ and each integer $k$, the modules $H_{k}(X, X-\{x\} ; L)$ and $H_{k}(X, X-\{y\} ; L)$ are isomorphic and finitely generated. The more recent work in [Br] applies to homogeneous ANR's and provides an alternative approach to that in $\left[\mathbf{B}_{\mathbf{1}}\right]$ in this specialized setting. The purpose of this note is to establish Bredon's result for a general principal ideal domain. The approach that is taken naturally breaks into two parts. The first involves an analysis of presheaves of $L$-modules for a commutative ring $L$, on completely metrizable spaces, that induce sheaves having mutually isomorphic finitely generated stalks. The conclusion is that a large class of such presheaves induce sheaves that are locally constant over a dense open set. The second, following the approach in $\left[\mathbf{B}_{\mathbf{1}}\right]$, restricts to the setting of homology presheaves with coefficient ring a principal ideal domain and combines the first result with a result from $\left[\mathbf{B}_{\mathbf{2}}\right]$ to conclude that a dense open subset of $X$ is a homology manifold and, since the stalks of the homology presheaf are assumed to be mutually isomorphic, $X$ itself is a homology manifold.

The analyses that follow differ from that in $\left[\mathbf{B}_{\mathbf{1}}\right]$ primarily in that it is done at the level of presheaves and sheaves (rather than specializing to homology presheaves and homology sheaves). A consequence of establishing the first step in the proof at the level of presheaves and sheaves is that the approaches, up to now, to settling the Bing-Borsuk Conjecture that a finite dimensional homogeneous ANR is a manifold (or at least a homology manifold) have not been sufficiently specialized to have

Received by the editors June 10, 1986 and, in revised form, May 1, 1987.

1980 Mathematics Subject Classification (1985 Revision). Primary 57P99.

Key words and phrases. Homology manifold, sheaf.

Supported in part by NSF grants. 
been successful. The point is that under the assumption that the stalks of the homology sheaf are finitely generated the critical result is valid at the level of sheaves while without the assumption of finite generation there are a variety of examples of sheaves for which the result fails.

The main result (namely, Theorem 1) of this note has useful generalizations and other applications. The reader is referred to $\left[\mathbf{D W _ { 1 }}\right]$ for an exposition and an additional application to homology manifolds. In addition, in $\left[\mathbf{D W _ { 2 }}\right]$ there is an application that yields estimates on the extent to which certain mappings can raise dimension.

We shall introduce definitions and notation as needed.

Presheaves inducing sheaves having mutually isomorphic finitely generated stalks. While the result in Theorem 1 is valid for presheaves and sheaves of modules over a commutative ring, the application in Theorem 2 makes use of a result from $\left[\mathbf{B}_{\mathbf{2}}\right]$ that requires the ring to be a principal ideal domain. For a presheaf $\mathbf{S}$ on a space $X$, we shall denote the inclusion induced homomorphism by $\mathbf{S}(U) \rightarrow \mathbf{S}(V)$. The stalk at a point $x$ is denoted by $\mathscr{S}_{x}$ and is equal to $\operatorname{dir} \lim \{\mathbf{S}(U): U$ is open and $x \in U\}$. For an open set $U$, point $x \in U$, and element $\alpha \in \mathbf{S}(U)$, the image of $\alpha$ in the stalk $\mathscr{S}_{x}$ is denoted by $\alpha_{x}$. The sheaf induced by $\mathbf{S}$ is denoted by $\pi: \mathscr{S} \rightarrow X$. Recall that as a set $\mathscr{S}$ is the disjoint union of the stalks $\mathscr{S}_{x}, x \in X, \pi$ "projects" each stalk $\mathscr{S}_{x}$ to the point $x$, and a basis for topology on $\mathscr{S}$ consists of sets of the form $\left\{\alpha_{x}: x \in U\right\}$ where $U$ is open and $\alpha \in \mathbf{S}(U)$.

A sheaf $\mathscr{S}$ on $X$ is a constant sheaf provided there is an $L$-module $G$ and a sheaf isomorphism $h: X \times G \rightarrow \mathscr{S}$ (that is, $G$ has the discrete topology, $h$ is a homeomorphism and, for each $x \in X$, the restriction of $h$ is a module isomorphism from $\{x\} \times G$ to $\mathscr{S}_{x}$ ). A sheaf $\mathscr{S}$ on $X$ is a locally constant sheaf provided each $x \in X$ is contained in an open set $U$ such that restriction of the sheaf $\mathscr{S}$ to $U$ is a constant sheaf over $U$.

A presheaf $\mathbf{S}$ is locally finitely generated provided, for each open set $U$ and $x \in U$, there is an open set $V, x \in V \subset U$, with $\operatorname{im}\{\mathbf{S}(U) \rightarrow \mathbf{S}(V)\}$ a finitely generated $L$-module.

THEOREM 1. Suppose $G$ is a finitely generated L-module, where $L$ is a commutative ring, and $X$ is a complete metrizable space. If $\mathbf{S}$ is a locally finitely generated presheaf on $X$ such that each stalk $\mathscr{S}_{x}$ is isomorphic to a fixed L-module $G$, then there is a dense open subset $W$ of $X$ such that the sheaf $\mathscr{S}$ induced by $\mathbf{S}$ restricts to a locally constant sheaf over $W$.

PROOF. For each $x \in X$ and $k>0$, let $U_{x, k}$ be the $2^{-k}$-neighborhood of $x$. If $n>m>0$ are integers let

$$
A_{m, n}=\left\{x \in X: \operatorname{im}\left(\mathbf{S}\left(U_{x, m}\right) \rightarrow \mathbf{S}\left(U_{x, n}\right)\right) \text { maps isomorphically onto } \mathscr{P}_{x}\right\} .
$$

ClaIM 1. For any integers $k$ and $q, X=\bigcup\left\{A_{m, n}: m>k, n>q\right\}$.

PROOF OF CLAIM 1. Given $x$ and $X$ choose a neighborhood $U$ of it such that $\mathbf{S}(U) \rightarrow \mathscr{P}_{x}$ is an epimorphism (recall that $\mathscr{P}_{x}$ is finitely generated). Take $m>k$ such that $U_{x, m} \subset U$. Now find $V$ such that $\operatorname{im}\left(\mathbf{S}\left(U_{x, m}\right) \rightarrow \mathbf{S}(V)\right)$ is finitely generated. The finitely generated submodule $I=\operatorname{im}\left(\mathbf{S}\left(U_{x, m}\right) \rightarrow \mathbf{S}(V)\right)$ maps onto $\mathcal{P}_{x}$ and the kernel of the map $I \rightarrow \mathcal{P}_{x}$ is finitely generated. Hence, we can find a neighborhood $V^{\prime} \subset V$ of $x$ such that $\operatorname{ker}\left(\mathbf{S}\left(U_{x, m}\right) \rightarrow \mathscr{P}_{x}\right)=\operatorname{ker}\left(\mathbf{S}\left(U_{x, m}\right) \rightarrow \mathbf{S}\left(V^{\prime}\right)\right)$. 
Now choose $n>q$ such that $U_{x, n} \subset V^{\prime}$. It is clear that $\operatorname{im}\left(\mathbf{S}\left(U_{x, m}\right) \rightarrow \mathbf{S}\left(U_{x, n}\right)\right)$ maps isomorphically onto $\mathscr{S}_{x}$.

Since $X$ is complete there exist $q>k>0$ such that $A_{k, q}$ is dense in an open nonempty set $P$ of $X$.

Claim 2. $P \subset\left\{x \in X: \mathbf{S}\left(U_{x, k+1}\right) \rightarrow \mathscr{S}_{x}\right.$ is an epimorphism $\}$.

ProOF OF Claim 2. Let $x \in P$. By Claim 1 there exist $n$ and $m, n>m$, with $m>k+1$ and $n>q+1$ such that $\operatorname{im}\left(\mathbf{S}\left(U_{x, m}\right) \rightarrow \mathbf{S}\left(U_{x, n}\right)\right)$ maps isomorphically onto $\mathscr{S}_{x}$. Choose $y \in P \cap A_{k, q} \cap U_{x, n}$. Then $U_{x, m} \subset U_{y, k}$ and $U_{x, n} \subset U_{y, q}$ so we have a commutative diagram

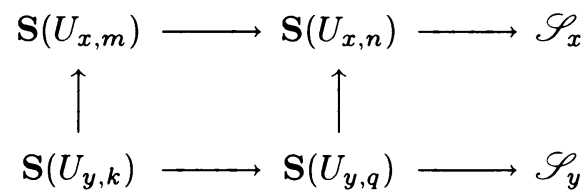

and $\operatorname{im}\left(\mathbf{S}\left(U_{y, k}\right) \rightarrow \mathbf{S}\left(U_{x, n}\right)\right) \subset \operatorname{im}\left(\mathbf{S}\left(U_{x, m}\right) \rightarrow \mathbf{S}\left(U_{x, n}\right)\right)$. Both these submodules of $\mathbf{S}\left(U_{x, n}\right)$ are isomorphic to $G$, as the latter maps isomorphically to $\mathscr{S}_{x}$ and the former maps isomorphically to $\mathscr{S}_{y}$. In fact the existence of this last isomorphism shows that $\operatorname{im}\left(\mathbf{S}\left(U_{y, k}\right) \rightarrow \mathbf{S}\left(U_{x, n}\right)\right)=\operatorname{im}\left(\mathbf{S}\left(U_{x, m}\right) \rightarrow \mathbf{S}\left(U_{x, n}\right)\right)$. (The property needed is that epimorphisms of isomorphic finitely generated modules over a commutative ring are isomorphisms [V, Proposition 1.2].) Therefore $\mathbf{S}\left(U_{y, k}\right) \rightarrow \mathscr{S}_{x}$ is an epimorphism and, since $U_{x, k+1} \subset U_{y, k}, \mathbf{S}\left(U_{x, k+1}\right) \rightarrow \mathscr{S}_{x}$ is an epimorphism.

Choose a point $x \in P$. By Claim $1, \operatorname{im}\left(\mathbf{S}\left(U_{x, m}\right) \rightarrow \mathbf{S}\left(U_{x, n}\right)\right)$ maps isomorphically onto $\mathscr{S}_{x}$ for some $n>m$ such that $m>k+1$ and $U_{x, m} \subset P$. If $y \in U_{x, n}$, then $U_{y, k+1} \supset U_{x, m}$ and Claim 2 implies that $\mathbf{S}\left(U_{x, m}\right) \rightarrow \mathscr{S}_{y}$ is an epimorphism. Therefore $\operatorname{im}\left(\mathbf{S}\left(U_{x, m}\right) \rightarrow \mathbf{S}\left(U_{x, n}\right)\right)$ maps isomorphically onto $\mathscr{S}_{y}$ (obviously the homomorphism is an epimorphism and both these modules are isomorphic to $G$ ).

Claim 3. $\mathscr{S} \mid U_{x, n}$ is constant.

PROOF OF Claim 3. Define $p: U_{x, n} \times \mathscr{S}_{x} \rightarrow \mathscr{S} \mid U_{x, n}$ as follows: if $y \in U_{x, n}$ and $\alpha \in \mathscr{S}_{x}$, we choose $\tilde{\alpha} \in \mathbf{S}\left(U_{x, m}\right)$ which maps onto $\alpha$ and then we set $p(y, \alpha)$ equal to the image of $\tilde{\alpha}$ in $\mathscr{S}_{y}$. The map $p$ is well defined because

$$
\operatorname{ker}\left(\mathbf{S}\left(U_{x, m}\right) \rightarrow \mathscr{S}_{y}\right)=\operatorname{ker}\left(\mathbf{S}\left(U_{x, m}\right) \rightarrow \mathbf{S}\left(U_{x, n}\right)\right)
$$

for $y \in U_{x, n}$. For an open set $V \subset U_{x, m}$ and $\alpha \in \mathbf{S}(V), p^{-1}\left(\left\{\alpha_{z}: z \in V\right\}\right)=V \times \mathscr{S}_{x}$ and, hence, $p$ is continuous. Similarly, for each $\alpha \in \mathbf{S}\left(U_{x, m}\right)$ and each open set $V \subset U_{x, n}$ the set of all images of $\alpha$ in $\mathscr{S}_{z}, z \in V$, is open in $\mathscr{S} \mid U_{x, n}$, and, hence, $p$ is an open map. Evidently, $p$ is one to one and onto and is a sheaf isomorphism.

Now define $W$ as $\left\{x \in X: \mathscr{S} \mid U_{x, n}\right.$ is constant for some $\left.n\right\}$. Obviously $W$ is open and $X^{\prime}=X-\operatorname{cl}(W)$ must be empty (otherwise we would apply Claims 1-3 to $\left.\mathscr{S} \mid X^{\prime}\right)$. This completes the proof of Theorem 1 .

Spaces with mutually isomorphic finitely generated local homology modules and homology manifolds. From now on we shall assume that all spaces are locally compact and metrizable. Singular homology theory is denoted $H_{k}(X ; L)$ where $L$ is the coefficient ring.

The symbol $\mathrm{HLC}_{L}$ is used to denote homologically locally connected with respect to singular homology with coefficient ring $L$; namely, $X$ is $\mathrm{HLC}_{L}$ provided, for each $x \in X$ and each neighborhood $U$ of $x$, there is a neighborhood $V \subset U$ of $x$ such that 
the inclusion induced homomorphism $\widetilde{H}_{k}(V ; L) \rightarrow \widetilde{H}_{k}(U ; L)$ is trivial for $k \geq 0(\widetilde{H}$ denotes reduced homology).

For a space $X, \mathbf{H}_{k}$ denotes the ( $k$-dimensional) homology presheaf on $X$ (with respect to the ring $L)$; that is, for each open set $U, \mathbf{H}_{k}(U ; L)=H_{k}(X, X-U ; L)$. The sheaf induced by $\mathbf{H}_{k}$ is denoted by $\mathscr{H}_{k}$.

A locally compact metrizable space $X$ is a homology $n$-manifold over $L$ provided it is homologically locally connected $\left(\mathrm{HLC}_{L}\right)$, has finite cohomological dimension with respect to $L$, and the stalks of its homology presheaf $\mathbf{H}_{k}$ are isomorphic to 0 for $k \neq n$ and isomorphic to $L$ for $k=n$.

A standard (and more general) notion of homology $n$-manifold uses Steenrod (equivalently, Borel-Moore) homology in place of singular homology. The analysis that follows applies equally in this setting, though there are minor differences. Details of such an extension can be found in $\left[\mathbf{D W} \mathbf{W}_{\mathbf{1}}\right]$.

THEOREM 2. Let $L$ be a principal ideal domain and let $X$ be a locally compact metrizable space that is homologically locally connected with respect to $L\left(\mathrm{HLC}_{L}\right)$ and has cohomological dimension $n$ with respect to $L$. If for any two points $x, y \in X$ and $k \leq n$, the modules $H_{k}(X, X-\{x\} ; L)$ and $H_{k}(X, X-\{y\} ; L)$ are isomorphic and finitely generated, then $X$ is a homology n-manifold over $L$.

PROOF. The assumption that $X$ has cohomological dimension $n$ assures that the presheaf $\mathbf{H}_{k}$ (hence, the sheaf $\mathscr{H}_{k}$ as well) is trivial for $k \geq n+1$. The strategy is to show that Theorem 1 applies to each of the presheaves $\mathbf{H}_{k}$. As this would assure the existence of a nonempty open set 0 of $X$ over which each $\mathscr{H}_{k}$ is constant. The homology sheaves being constant implies that 0 is a homology $n$-manifold, a fact that is recorded in $\left[\mathbf{B}_{\mathbf{2}}\right.$, Theorem 15.2] and that requires that $L$ is a principal ideal domain. (Alternatively, an exposition can be found in $\left[\mathbf{D W}_{\mathbf{1}}\right]$ that extracts the essential ingredients needed for this application; in particular, there is no need to use sheaves as coefficients.) Excision detects that $H_{k}(0,0-\{x\} ; L) \approx H_{k}(X, X-\{x\} ; L)$ and, consequently, the assumption that $H_{k}(X, X-\{x\} ; L)$ and $H_{k}(X, X-\{y ; L\})$ are isomorphic for $x, y \in X$ reveals that $X$ is a homology $n$-manifold.

We now turn our attention to showing that each $\mathbf{H}_{k}$ satisfies the hypotheses of Theorem 1.

It is a basic property of singular homology that

$$
\operatorname{dir} \lim \left\{H_{k}(X, X-U ; L): x \in U \text { and } U \text { open }\right\} \approx H_{k}(X, X-\{x\} ; L)
$$

Consequently, the different stalks of $\mathbf{H}_{k}$ are mutually isomorphic and finitely generated.

In order to show that $\mathbf{H}_{k}$ is locally finitely generated, we shall need to use the fact that if $\operatorname{cl}(K)$ is compact and is contained in the $\operatorname{int}(C)$ for a subset $C$ of $X$, then $\operatorname{im}\left\{H_{j}(K ; L) \rightarrow H_{j}(C ; L)\right\}$ is finitely generated (a proof based on the Mayer-Vietoris sequence is given in Appendix B). Given an open set $U$, with $\operatorname{cl}(U)$ compact, and $x \in U$, choose open sets $x \in V \subset W \subset U$ with $\operatorname{cl}(V) \subset W$ and $\operatorname{cl}(W) \subset U$, and choose compact sets $A, B$ and $C$ with $\operatorname{cl}(U) \subset$ int $A, A \subset$ int $B$ 
and $B \subset$ int $C$. Consider the diagram

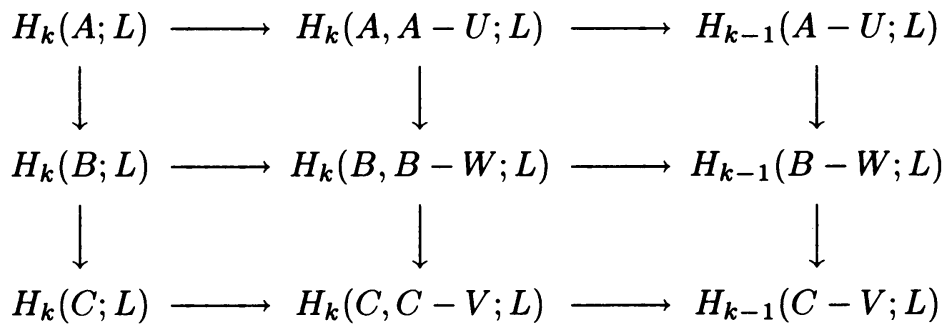

in which each of the horizontal sequences is part of the exact sequence of a pair. The observation at the beginning of the paragraph implies that both

$$
\operatorname{im}\left\{H_{k-1}(A-U ; L) \rightarrow H_{k-1}(B-W ; L)\right\} \quad \text { and } \operatorname{im}\left\{H_{k}(B ; L) \rightarrow H_{k}(C ; L)\right\}
$$

are finitely generated. It follows that $\operatorname{im}\left\{H_{k}(A, A-U ; L) \rightarrow H_{k}(C, C-V ; L)\right\}$ is finitely generated (see Appendix A). Since

$$
\begin{gathered}
\mathbf{H}_{k}(U ; L) \approx H_{k}(X, X-U ; L) \approx H_{k}(A, A-U ; L) \text { and } \\
\mathbf{H}_{k}(V ; L) \approx H_{k}(X, X-V ; L) \approx H_{k}(C, C-V ; L),
\end{gathered}
$$

we conclude that $\operatorname{im}\left\{\mathbf{H}_{k}(U ; L) \rightarrow \mathbf{H}_{k}(V ; L)\right\}$ is finitely generated.

A PPENDIX A. The following is an algebraic result that is used in the proof of Theorem 2 as well as in the proof given in Appendix B.

PROPOSITION. If in the commutative diagram

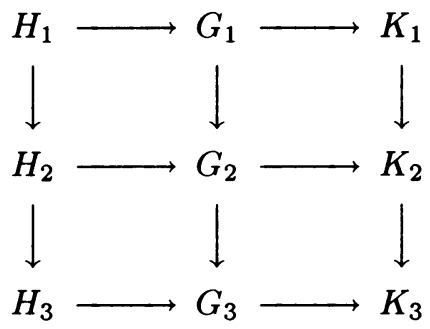

each row is exact (i.e., $\operatorname{im}\left\{H_{i} \rightarrow G_{i}\right\}=\operatorname{ker}\left\{G_{i} \rightarrow K_{i}\right\}$ for $i=1,2,3$ ) and both $\operatorname{im}\left\{K_{1} \rightarrow K_{2}\right\}$ and $\operatorname{im}\left\{H_{2} \rightarrow H_{3}\right\}$ are finitely generated, then $\operatorname{im}\left\{G_{1} \rightarrow G_{3}\right\}$ is finitely generated.

PROOF. Since $\operatorname{im}\left\{K_{1} \rightarrow K_{2}\right\}$ is finitely generated, we can specify elements $a_{1}, a_{2}, \ldots, a_{p}$ in $G_{2}$ such that $\operatorname{im}\left\{G_{1} \rightarrow K_{2}\right\}$ is generated by the images of $a_{1}, a_{2}, \ldots, a_{p}$ in $K_{2}$. Similarly, there are elements $b_{1}, b_{2}, \ldots, b_{q}$ in $G_{2}$ such that image of $H_{2}$ in $G_{3}$ is generated by the images of $b_{1}, b_{2}, \ldots, b_{q}$ in $G_{3}$. The claim is that the $\operatorname{im}\left\{G_{1} \rightarrow G_{3}\right\}$ is generated by the images of $a_{1}, a_{2}, \ldots, a_{p}$ and $b_{1}, b_{2}, \ldots, b_{q}$ in $G_{3}$. Let $g_{1} \in G_{1}$ and denote by $g_{2}$ and $g_{3}$ the image of $g_{1}$ in $G_{2}$ and $G_{3}$, respectively. There is a linear combination $\sum n_{i} a_{i}$ such that $g_{2}-\sum n_{i} a_{i}$ maps to 0 in $K_{2}$. Choose $h_{2} \in H_{2}$ such that $h_{2}$ maps to $g_{2}-\sum n_{i} a_{i}$ in $G_{2}$. There is a linear combination $\sum m_{i} b_{i}$ such that $\operatorname{im}\left(h_{2}\right)-\sum m_{i} b_{i}$ maps to 0 in $G_{3}$. It follows that the image of $\sum n_{i} a_{i}+\sum m_{i} b_{i}$ in $G_{3}$ is equal to $g_{3}$.

APPENDIX B. The next result is well known; the proof we present is particularly simple in that it uses only the Mayer-Vietoris sequence and the algebraic result from Appendix A. The reader can find essentially the same proof in [B, pp. 77-78]. 
THEOREM. Let $X$ be a locally compact metric space that is locally homologically connected with respect to a principal ideal domain $L\left(\mathrm{HLC}_{L}\right)$. If $\operatorname{cl}(A)$ is compact and $\operatorname{cl}(A) \subset \operatorname{int}(B)$, for subsets $A$ and $B$ of $X$, then $\operatorname{im}\left\{H_{k}(A ; L) \rightarrow H_{k}(B ; L)\right\}$ is finitely generated.

Proof. Clearly it suffices to consider the case with $A$ compact and $B$ open. By induction we assume that result is valid for $j \leq k-1$. Let $\mathscr{A}=\{C \subset B$ : $C$ is compact and is contained in an open set $N_{C} \subset B$ such that $\operatorname{cl}\left(N_{C}\right)$ is compact and $\operatorname{im}\left\{H_{k}\left(N_{C} ; L\right) \rightarrow H_{k}(B ; L)\right\}$ is finitely generated $\}$. Since $X$ is HLC, the collection $\mathscr{A}$ forms a cover of $B$. Consequently, the Theorem will follow once we show that $C_{1}, C_{2} \in \mathscr{A}$ implies that $C_{1} \cup C_{2} \in \mathscr{A}$. To that end specify open sets $U_{1}$ and $U_{2}$ with $C_{1} \subset U_{1}, C_{2} \subset U_{2}$ and $\operatorname{cl}\left(U_{1}\right) \subset N_{C_{1}}, \operatorname{cl}\left(U_{2}\right) \subset N_{C_{2}}$. Consider the diagram, whose rows come from the Mayer-Vietoris sequence,

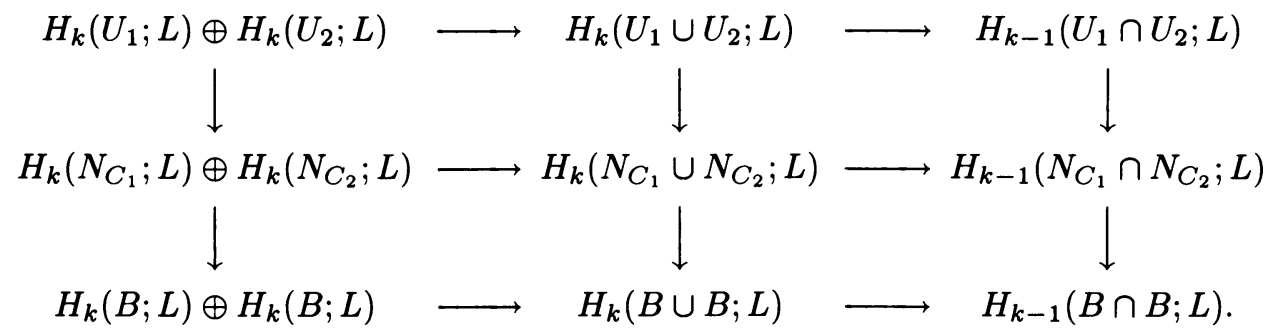

The result in Appendix A applies to show that $\operatorname{im}\left\{H_{k}\left(U_{1} \cup U_{2} ; L\right) \rightarrow H_{k}(B ; L)\right\}$ is finitely generated and, hence, $C_{1} \cup C_{2} \in \mathscr{A}$.

\section{REFERENCES}

[B1] G. Bredon, Generalized manifolds, Revisited, Proc. Georgia Conf. 1969 (J. C. Cantrell and C. H. Edwards, eds.), Markam, Chicago, Ill., 1970.

$\left[\mathbf{B}_{\mathbf{2}}\right]$, Sheaf theory, McGraw-Hill, New York, 1967.

[Br] J. Bryant, Homogeneous ENR's, preprint.

[B-M] A. Borel and J. C. Moore, Homology theory for locally compact spaces, Michigan Math. J. 7 (1960), 137-159.

[DW 1 J. Dydak and J. Walsh, Sheaves that are locally constant with applications to homology manifolds, Proc. Conf. Shape Theory and Geometric Topology, Dubrovnik, Yugoslavia, 1986.

$\left[\mathbf{D W}_{\mathbf{2}}\right] \ldots$, Estimates of the dimension of decomposition spaces, Lecture Notes in Math., vol. 1283, Springer-Verlag, 1987, pp. 65-87.

[M] W. S. Massey, Homology and cohomology theory, Marcel Dekker, New York, 1978.

[V] W. V. Vasconcelos, On finitely generated flat modules, Trans. Amer. Math. Soc. 138 (1969), 505-512.

Department of Mathematics, University of Tennessee, Knoxville, TennesSEE 37996

Department of Mathematics and Computer Science, University of CaliFORNIA, RIVERSIDE, CALIFORNIA 92521 